\section{OC1 CLINICAL AND MOLECULAR FEATURES OF TWENTY CHILDREN WITH HYPER-IGE SYNDROME CAUSED BY STAT3 GENE MUTATION IN MAINLAND CHINA}

Jiang Liping*, Xiang Qingqing. Children's Hospital of Chongqing Medical University, Chongqing, China

\subsection{6/archdischild-2019-epa.1}

Background and aims STAT3 gene mutation causes autosomal dominant hyper- IgE syndrome (AD-HIES)is a rare primary immunodeficiency disease. We described the clinical and molecular features of 20 AD-HIES patients in Mainland China.

Methods We diagnosed patients with AD-HIES on the basis of NIH score of $\geq 40$ points, described the clinical features, sequenced the STAT3 gene of 20 patients, and quantified Th17 cells in peripheral blood of 19 patients by flow cytometry.

Results Among the 20 patients (14 males and 6 females), the onset age ranged from 1 days to 6 months, and the diagnostic age ranged from 1 to 12 years. All patients (100\%) had characteristic manifestation of HIES with the range of NIH scores 41-77 points, including recurrent eczema and pneumonia, particularly high serum IgE levels and eosinophilia. Characteristic facial features (100\%), bone abnormalities, recurrent skin abscesses (85\%), lung abscesses (60\%), recurrent oral thrush $(55 \%)$ were also present in the studied patients. STAT3 hot mutations $\mathrm{V} 637 \mathrm{M}$ or $\mathrm{R} 382 \mathrm{~W} / \mathrm{Q}$ were identified in 12 patients (60\%), two novel heterozygous missense mutations (T620S and R609G) were identifiedin 2 patients respectively, and other mutations (C712G, S636F, Q469H, Y705H, V432M, c. $1139+5 \mathrm{G}>\mathrm{T})$ were identified in other 6 patients. The percentage of Th17 cells in CD4+ T cells (CD3+CD8IL-17A+/ CD3+CD8 cells) of 19 patients with STAT3 mutation was significantly decreased or deficient as compared with healthy controls.

Conclusions Children with repeated Staphylococcus aureus pneumonia, cold skin abscess, fungal infection, eczema, increased serum IgE level and eosinophil, should be alert to AD-HIES. Detection of Th17 cell by flow cytometry assist clinical diagnosis when the NIH score $\geq 40$ points. Further STAT3 gene analysis can confirmed the diagnosis.Early diagnosis and active prevention of infection can significantly improve the quality of life of patients.

\section{OC2 A NEW PEDIATRIC EDUCATIONAL MODEL: NEONATAL APPLICATION OF WORD CLOUD ANALYSIS AND SINGLE WORD SUMMARISATION}

Roy K Philip*. Graduate Entry Medical School (GEMS), University of Limerick, Limerick, Ireland. University Maternity Hospital Limerick (UMHL), Limerick, Ireland

10.1136/archdischild-2019-epa.2

Background Word cloud (WC) is the visual representation of a collection of text documents that uses various font sizes, colours, and spaces to arrange and depict significant words. WC generated from spontaneously articulated views by medical students as a single word summarisation (SWS) could assist in medical teaching. SWS could also assist pediatric teaching faculty to foster better student engagement. There is a paucity of published material in pediatric medical education using the WC analysis technologies.

Methods A descriptive study was conducted in the neonatal intensive care unit (NICU) of University Maternity Hospital
Limerick (UMHL) for five years from October 2012 to September 2017. One faculty member prospectively recorded the SWS of neonatology by medical students. Hospital audit committee approved study as a quality improvement project (QIP) in clinical teaching. Online WC generator under an open source license was used to compute the WC and an automated layout algorithm for positioning words without overlap was chosen.

Results Total of 268 SWS were generated from 268 graduate entry medical students who had neonatal rotations over five years. Words reflecting 'abstract' concepts predominated (example -rewarding) (182/268) compared to 'concrete' concepts (example- incubator) $(86 / 268)$ with $\mathrm{p}<0.005$. Numerical predominance with maximum repeatability was noted for the following fifteen words: intensive, ventilation, nutrition, emotional, high-risk, sepsis, premature, outcomes, risk, mortality, rewarding, nursing, feeding, sensitive and careful. Feedback data showed SWS and WC generation as unique 152/268 (56.7\%), stimulating $142 / 268(52.9 \%)$ and creative $130 / 268$ (48.5\%). Interestingly $72 / 268(26.8 \%)$ found it as a fun. A relatively small proportion found it not interesting 20/268 (7.4\%), boring 8/268 (3\%) and waste 4/268 (1.5\%). Power point slides of WC aided students to reflect on the visual impact of their chosen words.

Conclusion Visualization of medical student-generated SWS could stimulate reflection and clinical discussion. SWS could assist teaching faculty to foster better student engagement. This narrative teaching methodology with computer aided WC enhancement has the potential transferability to other clinical disciplines.

\section{OC3 A HOST-RESPONSE ASSAY DISTINGUISHES BETWEEN SIMPLE INFLUENZA PATIENTS AND INFLUENZA PATIENTS WITH BACTERIAL COINFECTION}

${ }^{1,2}$ Adi Klein*, ${ }^{3}$ Meital Paz, ${ }^{3}$ Kfir Oved, ${ }^{3}$ Asi Cohen, ${ }^{2,3,4}$ Ellen Bamberger, ${ }^{2,4}$ Isaac Srugo, ${ }^{2,4}$ Israel Potasman, ${ }^{3}$ Eran Eden, ${ }^{3}$ Liran Shani. ${ }^{1}$ Hillel Yaffe Medical Center, Hadera, Israel; ${ }^{2}$ Technion-Israel Institute of Technology, Haifa, Israel; ${ }^{3}$ Me Med, Tirat Carmel, Israel; ${ }^{4}$ BnaiZion Medical Center, Haifa, Israel

\subsection{6/archdischild-2019-epa.3}

Background Identifying bacterial coinfection in influenza patients can be difficult as the symptoms of simple influenza versus mixed infections are often similar, leading to antibiotic overuse. A new host-response assay (ImmunoXpert ${ }^{\mathrm{TM}}$ ) that integrates the levels of three proteins (TRAIL, IP-10, and CRP) was shown to exhibit high performance in distinguishing between bacterial and viral disease in two double-blind validation studies. Here we sought to evaluate its ability to differentiate between simple influenza and influenza with bacterial coinfection.

Material/Methods The study population included 653 febrile pediatric and adult patients prospectively recruited in the 'Curiosity' study. Patient etiology (simple viral versus mixed infection) was determined by unanimous expert adjudication based on comprehensive clinical, laboratory and radiological assessment. Influenza strains (A or B) were detected using multiplex PCR applied to nasal swabs (Seeplex-RV15). We compared the expert panel diagnosis with the assay that gives three possible outcomes: viral, bacterial (including viral with bacterial coinfection) or equivocal. An equivocal outcome does not provide diagnostic information and is observed in $\sim 10 \%$ of cases. 
Results Out of 653 patients, 51 had positive influenza detection and unanimous expert diagnosis: 44 simple viral infections and 7 influenza with bacterial coinfections (Figure 1). Antibiotics were prescribed to all 7 cases of influenza with bacterial coinfection and to 20/44 cases adjudicated as simple viral infections, indicating an overuse rate of $45 \%$. The assay correctly classified 40 of the 44 simple viral cases (out of the remaining 4, 2 were assigned viral with bacterial coinfection and 2 received equivocal outcomes) as well as 5 of the 7 viral with bacterial coinfection cases (the remaining 2 received equivocal outcomes) supporting the assay's potential to reduce antibiotic overuse 5 -fold (from $45 \%$ to $4 / 44=9 \%$, $\mathrm{P}<0.001$ ).

Conclusion The host-response assay can differentiate between simple influenza and influenza patients with bacterial coinfection, with potential to reduce antibiotic overuse. Utility studies are warranted to demonstrate that the assay can safely assist physicians in correct management of influenza patients

\section{OC4 THE QUALITY OF CHILD HEALTHCARE: A COMPARISON AMONG 30 EUROPEAN COUNTRIES}

${ }^{1}$ Fabrizio Pecoraro*, ${ }^{2}$ llaria Rocco, ${ }^{2}$ Barbara Corso, ${ }^{3}$ Oscar Tamburis, ${ }^{2}$ Nadia Minicuci, ${ }^{4}$ Daniela Luzi. 'A Institute for research on Population and Social Policies, National Research Council, Rome, Italy, Rome, Italy; '2Neuroscience Institute, National Research Council, Padua, Italy; ${ }^{3}$ Institute for research on Population and Social Policies, National Research Council, Rome, Italy; ${ }^{4}$ Institute for research on Population and Social Policies, National Research Council, Rome, Italy, Rome, Italy

\subsection{6/archdischild-2019-epa.4}

Background and goals Even though the conceptions and contents of quality of healthcare for children have substantially improved in the last decades, little is known about the routine adoption of measures used at national/regional level to evaluate childcare. This paper intends to investigate country practices in the evaluation of child healthcare providing insights on the priority posed in 30 European/EEA countries. It reports some of the main results achieved during the MOCHA (Models of Child Health Appraised) project.

Methods An ad hoc questionnaire was developed and sent to country-specific experts (CAs), appointed for each of the MOCHA's countries, to gather information on measures adopted to evaluate the quality of child care at country/ regional level. Results were analysed and compared on the basis of a two-level hierarchical map of domains that helped the comparison and also allowed the analysis on the breadth of child-centred evaluation of healthcare. The frequency of adoption of the whole set of measures was analyzed in order to investigate whether a similar behavior in terms of sparsity/ convergence is present across countries. Moreover, to identify whether a core set of common measures is adopted across countries, we analyzed measures reported by at least $25 \%$ of the CAs under the perspective of child-centeredness which may be also expressed by age ranges reported as variable of the measures and disease specificity.

Results Among the 27 CAs that completed the questionnaire, 23 countries reported to have a national/regional agency that assesses healthcare systems. 12 of them devote a specific part of the quality assessment to child health care, while the other countries integrate some child specific items within their health system evaluation. In total 352 measures were reported. The collected measures are not equally distributed among countries, as they range from a minimum of 6 measures in
Iceland to a maximum of 130 in the UK. Considering the analysis of core measures the most common one reported by 13 countries is the immunization rate, while a two other focal points can be identified in measures related to mortality and natality.

Conclusions The analyses confirmed a high variability across countries, both in number and type of measures adopted. Only a limited set of common measures, focused on immunization, mortality and hospitalization Only a limited set of common measures, focused on immunization, mortality and hospitalization, was identified, even if sparsely distributed among a restricted number of countries.

\section{OC5 'UP, AWAY AND OUT OF SIGHT' - HOW CHILDPROOF ARE CHILDPROOF MEDICATIONS?}

Aoife Cassidy*, Ronan Brady, Luke Keogh, Jean Donnelly. Our Lady's Children's Hospital Crumlin, Dublin, Ireland

10.1136/archdischild-2019-epa.5

Aims Despite numerous campaigns re storing medication safely away from children there are still many incidents of accidental poisoning each year. Parents often feel 'childproof' caps are $100 \%$ safe, even with inadequate storage. This study aims to assess how safe 'childproof' caps on medication are by determining if children of various ages can open these medication containers and if so, how quickly they can achieve this.

Methods Children aged up to 16 years old without severe physical or intellectual disabilities were included in this study. Data collection was obtained in the outpatient department while patients awaited their OPD appointment. The time taken for a child to open each 'childproof' medication container was recorded up to a maximum of two minutes. The age of the child and any relevant diagnosis was also recorded. Information on safe storage of medication was provided to parents.

Results Seventy-six children were included in this study to date. Ages ranged from 1 year to 16 years and were from a wide range of speciality clinics. Fourteen (18\%) children were able to open at least one container within 2 minutes (age range 4 to 16 years). All children 11 years and older were successful. All children aged 7 years and younger were unable to open the container, except one child who had been taught how to open his medications.

Twenty-five percent of females were able to open at least one medication bottle versus $14 \%$ of males, however there was a preponderance of females in the older age group. Of the children who were successful, the majority were able to open the container in less than 5 seconds. An incidental finding of this study was that in cases where the medication cap was not properly closed, most children were able to quickly open the container without difficulty.

Conclusion This study shows that the almost all children 7 years and younger were unable to open a childproof medication bottle in 2 minutes, however real-life data shows the majority of paracetamol and ibuprofen poisonings occur in children under 10 years old. Parents of young children often overestimate how safe 'childproof' medication containers are and underestimate what a child can do in a brief unsupervised period. Public awareness needs to be increased of the importance of proper closing and the safe storage of 'childproof' medications in order to minimize the risk of accidental poisoning. 